Revista de Antropología Social

ISSN: 1131-558X

http://dx.doi.org/10.5209/RASO 59434

\title{
¿Familia o trabajo? Procesos de parentalización entre au pairs latinoamericanas en el sur de Francia
}

\author{
Javier González Díez; ; Diego de la Torre Puente²
}

Recibido: 5 de julio de 2017 / Aceptado: 30 de octubre de 2017

Resumen. El artículo explora, a partir del caso de los au pairs, de qué manera sistemas de cuidado y asistencia familiar, que siempre se han fundado sobre "lazos fuertes", reaccionan frente a los fenómenos de desparentalización típicos de la segunda transición demográfica y al retroceso del Estado del bienestar. Basándose en una investigación etnográfica sobre la relación entre au pairs latinoamericanas y sus familias de acogida en la Región Provence-Alpes-Côte d'Azur del Sur de Francia, el artículo se interroga cómo las sociedades sur-europeas, caracterizadas por una ideología familista del cuidado y de la asistencia, gestionan la necesidad de incluir figuras externas en el ámbito de los cuidados familiares. El artículo evidencia cómo, a pesar del uso de un lenguaje de parentesco ficticio, la indeterminación de estas figuras deja abiertas múltiples posibilidades, que van desde una efectiva inclusión y parentalización por una parte, a una profesionalización y explotación laboral por otra.

Palabras clave: Desparentalización; au pair; cuidado y asistencia; familia; parentesco ficticio.

\section{[en] Family or work? Kinning Processes among Latin American au pairs in the South of France}

\begin{abstract}
Focusing on the case of au pairs, this article investigates how family care and assistance systems that have always been marked by "strong ties" react to processes of kin loss, typical of the second demographic transition, and to the retreat of the Welfare state. Based on an ethnographic research on the relationships between Latin American au pairs and their host families in the Provence-AlpesCôte d'Azur Region of the South of France, the article addresses the question of how South-European societies, characterised by a familistic ideology of care and assistance, face the need to include external figures in the field of family care. It shows how, despite the use of a fictive kinship language, the vague definition of the au pair figure leaves open multiple possibilities, ranging from effective inclusion and kinning to professionalization and labor exploitation.
\end{abstract}

Keywords: Kin loss; au pair; care and assistance; family; fictive kinship.

Sumario: 1. Introducción. 2. Au pairs, parentesco ficticio y servicio doméstico: enfoques teóricos y estado de la cuestión. 3. Au pairs latinoamericanas en el sur de Francia: una investigación. 4. Cuatro historias au pair. 5. La construcción de la relación entre au pairs y familias. 6. Conclusión: las contradicciones au pair, entre la construcción de un parentesco ideal y un servicio doméstico encubierto. 7. Referencias bibliográficas.

1 Doctor en Ciencias Humanas con especialización en Ciencias Antropológicas por la Universidad de Torino (Italia), actualmente es Profesor-Investigador en la Universidad Nacional de Educación (UNAE) del Ecuador, e-mail: javier.gonzalez@unae.edu.ec

2 Licenciado en Historia por la Universidad Autónoma de Zacatecas (México), actualmente es estudiante de la Maestría en Historia Contemporánea y Mundo Actual en la Universitat de Barcelona (España). e-mail: diego. delator@gmail.com 
Cómo citar: González Díez, J., De la Torre Puente, D. (2018). ¿Familia o trabajo? Procesos de parentalización entre au pairs latinoamericanas en el sur de Francia, en Revista de Antropología Social 27(1), 95-121.

\section{Introducción ${ }^{3}$}

En este artículo presentaremos los resultados de un proyecto de investigación sobre la relación entre jóvenes au pairs provenientes de Latinoamérica y sus familias de acogida en el sur de Francia. El artículo se inscribe en el panorama de estudios sobre las transformaciones de la familia en Europa meridional en las últimas décadas. En particular, nos interesa explorar de qué manera reaccionan los sistemas de cuidado y asistencia familiar, que siempre se han fundado sobre "lazos fuertes" (Reher, 1998), frente a los fenómenos de desparentalización típicos de la segunda transición demográfica, y al retroceso del Estado del bienestar. Nos interesa estudiar cómo las sociedades sur-europeas gestionan la necesidad de incluir figuras externas en el ámbito de los cuidados familiares, y cómo estas figuras están suspendidas entre dinámicas de inclusión de parentesco ficticio, por una parte, y de profesionalización, por otra (Zanotelli, 2010; Allen, Blieszner y Roberto, 2011).

El caso de las y los au pairs, una figura relativamente nueva en Europa meridional, se funda sobre un lenguaje de inclusión familiar, que prevé la incorporación temporal de sujetos externos a la red de parentesco para cubrir funciones desatendidas de ayuda y cuidado. En realidad, la falta de una tradición social o cultural alrededor de esta institución hace que el lenguaje familiar sea solo una metáfora (Carsten 2004), detrás de la cual la relación entre familias y au pairs puede asumir formas muy diferentes y contradictorias. La figura au pair puede ser, en efecto, potencialmente "parentalizable", pero también corresponder a un empleo doméstico encubierto. El lenguaje familiar puede originar una situación de inclusión, incorporación y reciprocidad, pero puede ser también una estrategia de control y explotación laboral, sobre todo cuando se aplica a sujetos vulnerables por su condición de migrantes.

En este artículo, intentaremos examinar las múltiples variantes que puede asumir la figura au pair y también su relación con la familia que la acoge, explorando qué elementos y qué factores condicionan la realización de un proceso de incorporación parental o bien de creación de una relación laboral encubierta.

\footnotetext{
Este artículo ha sido realizado en el marco de las actividades del Grupo de investigación "Familia, género y parentesco: perspectivas etnográficas y comparativas" del Departamento de Culturas, Política y Sociedad de la Universidad de Torino (Italia). Versiones preliminares del artículo fueron presentadas el 22/2/2017 en el ciclo de seminarios en Metodología de la Investigación Etnográfica del Doctorado en Ciencias Psicológicas, Antropológicas y de la Educación de la Universidad de Torino, y el 6/4/2017 en el ciclo de seminarios del Grupo de investigación AFIN de la Universitat Autónoma de Barcelona. Queremos expresar nuestro agradecimiento a Alessandro Gusman, Carlo Capello, Barbara Sorgoni, Bruna Álvarez Mora, por los comentarios y sugerencias avanzadas en el curso de esas presentaciones, y a Pier Paolo Viazzo por haber repetidamente leído y comentado las varias versiones del texto. Un agradecimiento enorme va también a todas y todos los au pairs que participaron en el proyecto, por la confianza con la que nos contaron sus experiencias. Si bien el artículo es fruto de una estrecha colaboración entre los autores, que han trabajado conjuntamente en todas las fases de la investigación y de la escritura, debe atribuirse a Javier González Díez la redacción de las secciones 1, 2, 3 y 6, mientras que a Diego de la Torre Puente la redacción de las secciones 4 y 5.
} 


\section{Au pairs, parentesco ficticio y servicio doméstico: enfoques teóricos y estado de la cuestión}

La crisis del Estado del bienestar en Europa meridional y el avance de la segunda transición demográfica han expuesto a las familias a quedar desatendidas de toda una serie de funciones que anteriormente aseguraban. En el origen de las dificultades de familias típicamente de lazos "fuertes" (Reher, 1998) encontramos principalmente la desparentalización, término con el que se designa la disminución del número medio de parientes por persona. Este fenómeno es, en primer lugar, el resultado del descenso de la natalidad que progresivamente ha caracterizado las transiciones demográficas en Europa, y ha supuesto una disminución cuantitativa del número de parientes y un debilitamiento de las redes tradicionales de parentesco (Solinas, 2004). En los países sur-europeos, caracterizados por regímenes de welfare débiles y de carácter "familista"" (Naldini y Jurado, 2008), esta disminución, unida a la crisis económica, ha reducido la capacidad de ayuda y solidaridad de la red familiar (Viazzo y González Díez, 2016).

Las dificultades de las familias en las tareas de cuidado se deben, también, a que la inclusión de las mujeres en el mercado laboral no ha sido acompañada por una transformación de los roles de género tradicionales en la familia mediterránea, ni tampoco por un aumento de la esfera de acción del Estado del bienestar (Segalen y Martial, 2013). Por este motivo, dentro de muchas familias las tareas domésticas y de cuidado de los hijos son consideradas aún de competencia de la mujer, incluso si esta trabaja (INSEE, 2012). Aunque ya ha sido evidenciado cómo las funciones de cuidado pueden ser frecuentemente delegadas a los abuelos (Attias-Donfut y Segalen, 1998), existen muchos casos en los que diversos factores alejan a las parejas de sus padres, dejándoles descubiertos en las funciones de cuidado de los hijos.

Las ciencias sociales han subrayado en los últimos años cómo la desparentalización es un fenómeno más global, y numerosos estudios han vuelto a explorar las estrategias que se ponen en acción "desde abajo" para conseguir cubrir las funciones desatendidas por la falta de parientes. Muchos estudios han empezado a considerar las formas de "parentesco ficticio", estudiadas por los antropólogos en varias partes del mundo hasta los años 70 y después parcialmente caídas en el olvido ${ }^{6}$. Estas formas prevén la inclusión en la red de parentesco de sujetos externos desde un punto de vista "bio-legal" , que vienen asimilados al estatus de parientes no solo nominalmente, sino también, y sobre todo, desde un punto de vista de los deberes y expectativas. Los estudios clásicos de antropología se han concentrado sobre todo en las formas más institucionalizadas y ritualizadas de parentesco ficticio, como el compadrazgo latinoamericano o los sistemas de fraternidad africanos, pero disminuyeron a partir de los años 80 con el llamado "paréntesis" en el estudio del parentesco. Con el surgimiento de los "nuevos estudios de familia", a finales de los años 90, el parentesco

4 Con el término "familista" indicamos la propensión a favorecer las solidaridades familiares respecto a los lazos sociales más generales.

5 Con el término de "parentesco ficticio" nos referimos convencionalmente a todas esas formas de relacionalidad que constituyen una extensión y re-interpretación del llamado parentesco "bio-legal". Este término se ha afirmado recientemente en las ciencias sociales (Nelson 2014) sin que su uso quiera suponer que exista efectivamente un parentesco "verdadero" contrapuesto a uno "ficticio".

6 Entre las obras que reseñan la mayor parte de esos trabajos, Pitt Rivers (1968).

7 Separamos el término, para distinguir las diferentes concepciones de parentesco, de Allen, Blieszner y Roberto (2011). 
ficticio ha vuelto a ser objeto de estudio por parte de la antropología y de las otras ciencias sociales, como ejemplo interesante y extremo de los lenguajes que pueden asumir las formas de "relacionalidad" y "parentalización" (Carsten, 2004), que permiten salir de los esquemas clásicos y euro-céntricos que representaban el parentesco fundado en la sangre o en la genética (Meillassoux, 2000; Remotti, 2008; Sahlins, 2013). Los estudios recientes han revelado cómo las formas institucionalizadas de parentesco ficticio han sobrevivido transformándose y adquiriendo nuevos matices (Jelm, 2010; González Díez y Viazzo, 2016), pero también cómo al mismo tiempo nacen formas más fluidas, informales y poco codificadas en el contexto europeo y norte-americano. Formas de inclusión de no-parientes en las redes de parentesco por necesidad de solidaridad y asistencia han sido observadas y estudiadas en Estados Unidos entre las comunidades afro-americanas (Chatters, Taylor y Jayakody, 1994), entre la población anciana de las clases sociales inferiores (Allen, Blieszner y Roberto, 2011), entre los migrantes latinos (Ebaugh y Curry, 2000) y entre las nuevas familias homosexuales (Murraco, 2006). Formas análogas, aunque basadas en un sostén moral más que material, han sido observadas también en Europa, en el contexto holandés (Voorpostel, 2013) y británico (Allan, 2008). Estos estudios se han concentrado sobre los procesos de "parentalización" de personas de forma voluntaria y afectiva, es decir, a partir de relaciones de amistad.

Recientemente, sin embargo, varios estudios han empezado a prestar atención también a las relaciones de parentalización que nacen de relaciones no de amistad sino de empleo, en particular ligadas al cuidado de los ancianos o de los niños. La posibilidad de que cuidadores y cuidadoras profesionales de ancianos sean incluidos, de nombre y de hecho, en una red familiar ha sido notada y estudiada en Estados Unidos (Karner, 1998) pero también en el contexto sur-europeo, en particular en Italia (Baldassar, Ferrero y Portis, 2017). Respecto al cuidado de los niños, existen varios estudios sobre las dinámicas de parentalización de las niñeras, siempre en Estados Unidos (Macdonald, 2010) y en el contexto sur-europeo (Zanotelli, 2010). Las figuras profesionales ligadas al cuidado familiar son candidatas ideales para la parentalización, pues comparten toda una serie de espacios y situaciones de intimidad, así como desarrollan funciones de cuidado y asistencia consideradas comúnmente típicas de los parientes (Kauffman, 1996). Sin embargo, su condición está llena de ambigüedades, pues existen profundas asimetrías de poder entre empleadores y empleadas que condicionan la relación, amplificadas por la vulnerabilidad en la que muchas de ellas se encuentran por su condición migrante ${ }^{8}$.

Entre las figuras objeto de dinámicas de parentalización, las au pair adquieren un interés particular, pues se colocan en una posición que ha sido definida en varias ocasiones como "marginal" (Búriková y Miller, 2010) o ambigua (Çanga, 2015). Los y las au pair no son considerados oficialmente figuras profesionales, sino más bien son presentados como jóvenes estudiantes que efectúan un intercambio de tipo

8 Numerosos estudios (Ehrenreich y Hochschild, 2003) analizan las asimetrías de poder en las relaciones de género y de empleo, avanzando la idea de una "feminización" de determinados trabajos, los cuales, independientemente de que sean realizados por personas de género masculino o femenino, reproducen relaciones subordinadas a un poder patriarcal. Generalmente, los trabajos en el área del cuidado o del empleo doméstico son considerados "feminizados" (Parreñas, 2001) y esto justifica por tanto el hecho de que se hable colectivamente de "las niñeras" o "las au pair". Siguiendo esta perspectiva — que se desmarca de la norma gramatical tradicional- en nuestro artículo haremos recurso a categorías gramaticalmente femeninas para remarcar la dimensión socialmente feminizada y subordinada del colectivo que realiza esas tareas. 
lingüístico y cultural a través de la inserción en una familia. De hecho, una au pair se encuentra en la condición de efectuar labores consideradas al mismo tiempo propias de la familia, pero también de las figuras profesionales externas, es decir las niñeras, y en muchas ocasiones también típicas del empleo doméstico.

La figura de la au pair, como la conocemos en la actualidad, nace hacia 1920 (Cuartas Villa, 2014), y es regulada en Europa a partir de 1969 a través de un Acuerdo europeo sobre las personas Au Pair ${ }^{9}$ firmado por 13 países europeos ${ }^{10}$. Aunque se trate por tanto de una figura relativamente antigua, que proviene de una tradición histórica donde el cuidado infantil estaba delegado por las clases altas a nodrizas, nanas y niñeras, es a partir de la última década del siglo XX que el fenómeno se ha incrementado de manera considerable. El Acuerdo Europeo establece que «les personnes placées au pair constituent une catégorie spécifique tenant à la fois de l'étudiant et du travailleur, sans entrer pour autant dans l'une ou l'autre de ces catégories". El Acuerdo es muy breve y sintético, y define la experiencia au pair como "l'accueil temporaire, au sein de familles, en contrepartie de certaines prestations" (art. 2). La au pair "reçoit nourriture et logement de la famille d'accueil" (art. 8) y en cambio "fournit à la famille des prestations consistant en une participation à des tâches familiales courantes" (art. 9). Si bien se indica un límite horario cotidiano a estas prestaciones ( 5 horas), no se indica en absoluto en qué tendrían que consistir las "tareas familiares corrientes", así como en qué horarios tendrían que ser realizadas, o a través de qué modalidades. Al mismo tiempo, se indica genéricamente que el o la au pair tiene derecho a recibir, como "propina" - no salario - "une certaine somme dont le montant et la périodicité de versement seront déterminés par l'accord visé à l'article 6 [acuerdo privado entre au pair y familia acogedora]" (art.8), que normalmente en Francia consiste en unos 80 euros por semana, pero que es dejado a la discreción de los contrayentes. Como podemos apreciar, la definición legislativa de la figura es extremamente vaga, y las legislaciones nacionales de los países que han ratificado el tratado no hacen otras especificaciones ${ }^{11}$. Esto es lo que permite que la figura au pair sea interpretada de facto de maneras muy diferentes, que oscilan entre el ser considerada/tratada como una componente temporal de la familia o como alguien de trabajo doméstico.

La ambigüedad de la figura au pair es señalada por buena parte de los estudios existentes, que reportan "las múltiples fachadas de las au pairs" (Durin, 2014), insistiendo sobre todo en la dimensión retórica que casi siempre asume la proclamación de pertenencia a la familia (Hess y Puckhaber, 2004; Cox, 2007; Bikova, 2008). Frente a un discurso oficial que, como hemos visto, define la "experiencia au pair" de tipo familiar, se ha denunciado cómo esta experiencia se revela, en cambio, en muchos casos como una condición de servicio doméstico enmascarado y explotado.

Consejo de Europa, Accord européen sur le placement au pair, Strasbourg, 24.XI.1969, http://www.coe.int/it/ web/conventions/full-list/-/conventions/rms/0900001680072357.

10 El acuerdo fue promovido por el Consejo de Europa, por lo que ha sido firmado también por países no pertenecientes a la Unión Europea, como Suiza, o a punto de salirse de ella, como Gran Bretaña. El tratado no menciona ni alienta por tanto una política común de visas entre los países acogedores, creando de hecho numerosas disparidades según las relaciones bilaterales que haya entre los países de origen de las au pairs y los países de acogida.

11 Las legislaciones nacionales normalmente se limitan a traducir o reportar sin grandes transformaciones el Acuerdo Europeo. Cfr. el caso de Francia, Décret n71-797 du 20 septembre 1971 PORTANT PUBLICATION DE L'ACCORD EUROPEEN SUR LE PLACEMENT AU PAIR, FAIT A STRASBOURG LE 24-11-1969, https:// www.legifrance.gouv.fr/affichTexte.do?cidTexte=JORFTEXT000000336159 
Anderson (2007), Mellini, Yodanis y Godenzi (2007), Muñoz Rodríguez (2014) y Cuartas Villa (2014) proponen contemplar la figura au pair dentro de la perspectiva de las "sirvientas globales" (Parreñas, 2001; Ehrenreich y Hochschild, 2003), evidenciando cómo la condición de la migración determina relaciones de poder asimétricas que desembocan muchas veces en explotación. En esta óptica, la figura au pair sería el resultado reciente de la historia del servicio doméstico europeo y colonial (Durin, 2014), pues en efecto las ideas de clase social y de "raza", junto a la subordinación de género, juegan un papel muy importante en su definición (Newcombe, 2004; Anderson, 2007; Durin, 2015). Pero, aún sin negar estas dimensiones de la experiencia au pair, hay quien señala su cercanía a experiencias históricas típicas del Norte de Europa, como el intercambio de jóvenes entre familias en los siglos XVIII-XIX (Sarti, 1999; Bikova, 2008; Çanga, 2015), que dejan abierta la puerta a la idea de que las y los au pairs puedan ser, efectivamente, parte de dinámicas de construcción del parentesco ficticio.

El caso de las au pairs es interesante, pues en él se mezclan dimensiones diferentes; por una parte, registros retóricos que proclaman la idea de parentesco ficticio como presupuesto a la realización de la experiencia; por otra, dimensiones materiales de relaciones de poder y de asimetría que pueden llegar a tomar varias direcciones, desde una inclusión familiar percibida como efectiva, a una exclusión que se convierte en explotación laboral encubierta. Entre las y los au pairs nos encontramos con una parentalización lingüística y retórica a priori, que en algunas ocasiones puede llegar a realizarse de facto, pero que en muchas otras se trasforma, en cambio, en una profesionalización de la figura que llega a perder sus elementos definitorios de fachada.

\section{Au pairs latinoamericanas en el sur de Francia: una investigación}

Numerosos estudios (Régnier-Lollier, 2015) han revelado la particularidad de Francia en el contexto europeo desde el punto de vista de los sistemas demográficos, de familia y parentesco, de las políticas sociales y de las ideas de acogida de los migrantes. En el marco de las macro-regiones que los estudiosos han intentado reconstruir en el ámbito europeo, Francia presenta muchas veces características socio-culturales intermedias entre la situación de los países de Europa noroccidental y la de los países mediterráneos.

En el panorama demográfico, por ejemplo, Francia ha mantenido unos niveles de natalidad intermedios entre el Norte y el Sur de Europa: si bien en descenso, la natalidad aún tiene valores bastante altos que han ayudado a mantener el saldo demográfico en activo en los últimos años. La particularidad de esta natalidad es que en realidad corresponde a una situación donde la nupcialidad es extremamente baja y las relaciones de convivencia han superado a las uniones matrimoniales: en 2010 se estima que casi el $60 \%$ de los niños franceses nacía de parejas no casadas (Segalen y Martial, 2013). Desde un punto de vista socio-cultural, la sociedad francesa ha experimentado el surgimiento de nuevas formas de convivencia - uniones libres, familias truncadas o recompuestas - con bastante adelanto respecto a los países del sur de Europa (Attias-Donfut, Lapierre y Segalen, 2002).

La fragmentariedad de las formas familiares se ve acompañada por una alta tasa de empleo femenino que caracteriza la experiencia de la maternidad desde sus ini- 
cios: más del $62 \%$ de las madres con niños de menos de 6 años trabajan, y este porcentaje sube a entre 76 y $86 \%$ si los hijos son mayores de seis años (Moschion, 2009). La alta presencia de niños y la tasa de empleo femenino más alta de Europa no se han visto compensadas por un incremento de las estructuras de cuidado como guarderías, que en Francia son pocas y que acogen solo a poco más del 10\% de los niños menores de tres años. El sistema de welfare se parece en esto más al modelo de los países del sur de Europa, según un modelo de familiarización del cuidado presentado como un "libre choix du mode de garde" (Segalen y Martial, 2013). Las madres francesas prefieren en su gran mayoría un modelo de cuidado de los hijos privado y familiar, y, como en el sur de Europa, esta elección responde ya sea a las limitaciones del welfare, o a valores socio-culturales que evidencian la presencia de "lazos fuertes" hacia los hijos más de tipo mediterráneo que norte-europeo. En este contexto, la ayuda de los abuelos no parece seguir las mismas pautas del sur de Europa y, pese a ser de todas formas relevante, no llega a cubrir las necesidades de asistencia y cuidado de las madres.

La particularidad del sur de Francia respecto a otras áreas del país es, en primer lugar, la de ser una región más mediterránea desde el punto de vista de las estructuras socio-demográficas familiares, pero al mismo tiempo la de presentar particulares trazos norte-europeos. La región de la Costa Azul, en efecto, ha sido objeto en las últimas décadas de una fuerte migración italiana y española y, más recientemente, de migraciones internas desde el norte de Francia. Si las familias de origen migrante sur-europeo ya están instaladas desde hace varias generaciones y entre ellas encontramos con más facilidad la idea de "lazos fuertes", quien proviene del norte está frecuentemente alejado de sus redes de parentesco y tiene valores y actitudes bastante diferentes respecto a la interacción con los familiares. El panorama que se presenta es pues el de una sociedad con características mixtas de las dos macro-regiones de Europa occidental, en presencia de un Estado del bienestar relativamente débil y con orientación familista (Régnier-Lollier, 2015).

Nuestra investigación se ha localizado fundamentalmente en el Sur de Francia, y se ha realizado entre au pairs provenientes de América Latina. El trabajo de campo de esta investigación se ha llevado a cabo entre los meses de abril y noviembre del 2016. En esta fase realizamos 12 entrevistas directas, y recogimos dos relatos de vida. Conjuntamente, Diego de la Torre participó activamente en el proyecto au pair desde septiembre de 2015 a septiembre de 2016 como observador participante, por lo que en este artículo se integran también observaciones basadas en su trabajo como au pair ${ }^{12}$. El método de recolección de información fue principalmente la entrevista semi-estructurada, donde a los participantes se les pedía que se presentaran y respondieran a algunas preguntas de base. Generalmente se les dio la posibilidad de hablar libremente. Las preguntas de base fueron relativas a datos generales de cada

12 Tenemos que reconocer que nos hubiese gustado también entrevistar a algunas familias y a miembros de las agencias, pero no nos fue posible. Las agencias generalmente no son nada disponibles al diálogo, y a pesar de haber contactado a varias de ellas, no logramos que nos concedieran ni un encuentro. En el caso de las familias, nos ha sido difícil encontrar nuevas, pues siguiendo recomendaciones éticas de otros investigadores (Durin 2014) hemos decidido no contactar a las familias de las y los au pairs entrevistados, como forma de tutela y protección hacia ellos. Habiéndonos concentrado en la figura au pair, no hemos encontrado por tanto familias adecuadas, pues estaban todas descartadas a priori. Por tanto, hemos decidido dejar el contacto con las familias para una fase futura de la investigación. 
au pair, datos de la familia de acogida, tareas y horarios, descripción del espacio de convivencia, relación con la familia, eventuales evaluaciones y episodios.

El grupo seleccionado (tabla 1) estaba ubicado en el sur de Francia, principalmente la Costa Azul, salvo cuatro casos donde, al principio las au pairs comenzaron en el norte de Francia y después cambiaron de familia al sur de Francia, y otro, que realizó dos estancias con 2 familias del centro de Francia. Las edades rondan entre los 19 y los 28 años, y se trata de 11 mujeres y 3 hombres ${ }^{13}$ que realizaron sus experiencias entre 2014 y 2016. Los países de origen son Colombia (7 mujeres), México (4 mujeres y 1 hombre), Costa Rica (1 hombre) y Brasil (1 hombre). Si bien el nivel escolar es bastante parecido, sus condiciones socio-económicas son bastante diferentes, oscilando entre clase media-alta y media-baja.

Tabla 1. Au pairs que han participado en la investigación

\begin{tabular}{|r|l|c|c|c|}
\hline N. & Nombre* & Edad** & Proveniencia & Tipo de estudios \\
\hline 1 & Margarita & 27 años & México & Estudios de grado \\
\hline 2 & Dolly & 24 años & Colombia & Estudios de grado \\
\hline 3 & Marta & 26 años & Colombia & Estudios de grado \\
\hline 4 & Dolores & 26 años & México & Educación superior \\
\hline 5 & Alejandra & 24 años & Colombia & Estudios de grado \\
\hline 6 & Pamela & 27 años & Colombia & Estudios de grado \\
\hline 7 & Ricardo & 20 años & Costa Rica & Educación superior \\
\hline 8 & Pablo & 25 años & Brasil & Estudios de grado \\
\hline 9 & Lucy & 25 años & Colombia & Estudios de grado \\
\hline 10 & Alfonso & 23 años & México & Estudios de grado \\
\hline 11 & Marina & 24 años & Colombia & Educación superior \\
\hline 12 & Eva & 26 años & Colombia & Educación superior \\
\hline 13 & Guadalupe & 20 años & México & Estudios de grado \\
\hline 14 & Ana & 19 años & México & Estudios de grado \\
\hline
\end{tabular}

* Por razones de privacidad, todos los nombres son apodos inventados por nosotros.

** Las edades se refieren al momento del comienzo de la experiencia au pair a la llegada a Francia

Naturalmente este grupo no tiene ninguna pretensión de representatividad estadística, pues se llegó a los participantes a través de una técnica de tipo "bola de nieve" fundada al comienzo sobre la red de contactos de Diego de la Torre. Las au pairs en el Sur de Francia no provienen exclusivamente de América Latina, y en el curso de la investigación entramos en contacto con au pairs provenientes de otros países como Alemania, Italia, España, Polonia, Rusia e Indonesia. Decidimos concentrarnos solo sobre las que provenían de América Latina para evitar el riesgo de

13 La menor presencia de hombres au pairs se puede deber a la percepción de esta actividad como "femenina" ( $c f r$. nota 8), por pertenecer al ámbito de los cuidados y de las actividades domésticas. Cuando las familias buscan alguien que se ocupe de sus hijos, casi de manera subconsciente buscarán una mujer. En su experiencia de observador participante Diego de la Torre encontró que algunas familias ni siquiera pensaban en la posibilidad de un au pair hombre, porque no se les había ocurrido. 
tener experiencias demasiado diferentes y poco comparables entre sí. Sin querer culturalizar y esencializar una idea de América Latina, hemos considerado que este macro-origen geográfico común, así como la condición de migrante - que, unida al género, determina el estatus social de todas ellas en términos de vulnerabilidad y subalternidad - nos ayudan a definir y circunscribir una serie de experiencias que se insertan en un horizonte de ideas sociales - sobre la familia, la sociabilidad, la educación, el cuidado, la vida cotidiana y la habitación, entre las muchas - que a pesar de su diversidad y pluralidad interna, seguramente tienen muchas más semejanzas entre sí que con quienes provienen de otras áreas geográficas. El grupo se caracterizaba, por tanto, por situaciones y experiencias muy diferentes, pero se puede trazar un perfil muy general del conjunto:

Agencias: Sólo 7 de las 14 au pairs entrevistadas llegaron a Francia por medio de una agencia, y de estas dos la abandonaron en el momento de cambiar de familia. Unas 6 au pairs llegaron a Francia por contacto directo con las familias. En varios casos familias y au pairs se conocieron gracias a la intermediación de alguna au pair precedente o gracias a redes de amistades comunes, en otros casos a través de búsquedas en redes sociales especializadas. Las agencias, como veremos, no parecen tener un papel relevante en la experiencia au pair, simplemente facilitan la documentación burocrática, pero en ningún caso nos parece que hayan hecho algo para preparar a au pairs y familias para el encuentro. Prácticamente en todos los casos de problemas entre au pairs y familias que hemos registrado, las agencias tuvieron un papel nulo.

Los horarios: las actividades au pair estaban basadas en el horario escolar francés, por lo que regularmente comenzaban a las $7 \mathrm{~h}$ para dar el desayuno y preparar a los niños para la escuela. Disponían de tiempo libre hasta las 15 o $16 \mathrm{~h}$. Generalmente las mañanas estaban ocupadas en cursos de francés, pero por lo menos unas 6 au pairs abandonaron estos cursos durante su experiencia. Por la tarde estaban con los niños desde las 16 hasta las $20 \mathrm{~h}$, que era después de la hora de la cena, cuando los padres habían regresado. Los miércoles, generalmente, el trabajo iniciaba a mediodía que es cuando finaliza la escuela ese día hasta las $20 \mathrm{~h}$.

Las familias: el grupo de 14 au pairs recorrieron un total de 23 familias (tabla 2). De estas, 14 se encontraban en la Costa Azul, una en otra localidad del sur de Francia, 3 en el centro de Francia, 4 en el Norte y una en Córcega. Sólo 7 au pairs de 14 estuvieron en una sola familia, las otras 7 tuvieron de 1 a 4 cambios de familia. En dos situaciones las au pairs entrevistadas habían vivido en la misma familia en fases sucesivas: Ana y Ricardo (familia 9), Eva y Lucy (familia 18). La mayoría de las familias tenía unos 2-3 hijos de edades variables, aunque principalmente en edad infantil ${ }^{14}$. Por lo menos 14 familias ya habían tenido au pairs, mientras que 5 estaban en su primera experiencia. Sabemos también que por lo menos 10 familias contaban con la ayuda de una femme de ménage para las tareas domésticas, que solo 4 tenían la posibilidad de tener la ayuda de los abuelos y 2 familias utilizaban también los servicios de una niñera.

14 Casi la mitad de los niños y niñas objeto de cuidado por parte de las au pairs que participaron en nuestra investigación era de edad inferior a 5 años, seguidos por el grupo de edad de 6 a 10 años; solo una minoría tenían edades superiores a los 11 años. 
Tabla 2. Esquema de las familias en relación a cada au pair

\begin{tabular}{|c|c|c|c|c|c|c|c|c|}
\hline N. & Nombre & Familia & $\begin{array}{c}\text { Lugar } \\
\text { en } \\
\text { Francia }\end{array}$ & Meses & Componentes & $\begin{array}{c}\text { Au pairs } \\
\text { en la } \\
\text { familia }\end{array}$ & $\begin{array}{c}\text { Otras } \\
\text { figuras } \\
\text { de ayuda } \\
\text { familiar }\end{array}$ & $\begin{array}{l}\text { Agen- } \\
\text { cia }\end{array}$ \\
\hline \multirow{3}{*}{1} & \multirow{3}{*}{ Margarita } & 1 & Norte & 3 & $\begin{array}{l}\text { Madre, Padre, } 3 \text { hijos }(11,8, \\
5 \text { años })\end{array}$ & $12 \mathrm{a} A \mathrm{P}$ & abuelos & sí \\
\hline & & 2 & Centro & 1 & Madre, una hija (3 años) & & & sí \\
\hline & & 3 & $\begin{array}{l}\text { Costa } \\
\text { Azul }\end{array}$ & 6 & $\begin{array}{l}\text { Madre, Padre, } 3 \text { hijos }(14,5, \\
4 \text { años })\end{array}$ & & empleada & sí \\
\hline \multirow{3}{*}{2} & \multirow{3}{*}{ Dolly } & 4 & Norte & 5 & $\begin{array}{l}\text { Madre, Padre, } 2 \text { hijos (14, } \\
11 \text { años) }\end{array}$ & & & sí \\
\hline & & 5 & $\begin{array}{l}\text { Costa } \\
\text { Azul }\end{array}$ & 16 & $\begin{array}{l}\text { Madre, Padre, } 3 \text { hijos (14, } \\
11,9 \text { años) }\end{array}$ & 3a AP & empleada & sí \\
\hline & & 6 & Córcega & 1 & Madre, Padre, 1 hija (8 años) & $11 \mathrm{a} \mathrm{AP}$ & & sí \\
\hline 3 & Marta & 7 & $\begin{array}{l}\text { Costa } \\
\text { Azul }\end{array}$ & 10 & $\begin{array}{l}\text { Madre, Padre, } 2 \text { hijos (1 año, } \\
3 \text { meses) }\end{array}$ & 1a AP & empleada & sí \\
\hline 4 & Dolores & 8 & $\begin{array}{l}\text { Costa } \\
\text { Azul }\end{array}$ & 11 & $\begin{array}{l}\text { Madre, Padre, } 3 \text { hijas }(12,6, \\
3 \text { años) }\end{array}$ & $8 \mathrm{a} A \mathrm{P}$ & empleada & no \\
\hline 5 & Alejandra & 9 & $\begin{array}{l}\text { Costa } \\
\text { Azul }\end{array}$ & 9 & $\begin{array}{l}\text { Madre, Padre, } 3 \text { hijos }(11,9, \\
5 \text { años })\end{array}$ & $2 \mathrm{a} A P$ & & no \\
\hline \multirow{2}{*}{6} & \multirow{2}{*}{ Pamela } & 10 & Norte & 8 & $\begin{array}{l}\text { Madre, Padre, } 2 \text { hijos }(6,3 \\
\text { años })\end{array}$ & 1a AP & & no \\
\hline & & 11 & $\begin{array}{l}\text { Costa } \\
\text { Azul }\end{array}$ & 15 & $\begin{array}{l}\text { Madre, Padre, } 3 \text { hijos }(6,3, \\
1 \text { año })\end{array}$ & 1a AP & empleada & no \\
\hline 7 & Ricardo & 12 & $\begin{array}{l}\text { Costa } \\
\text { Azul }\end{array}$ & 9 & $\begin{array}{l}\text { Madre, Padre, } 4 \text { hijos }(21,6, \\
3,3 \text { años })\end{array}$ & 4o AP & & sí \\
\hline \multirow{5}{*}{8} & \multirow{5}{*}{ Pablo } & 13 & $\begin{array}{l}\text { Costa } \\
\text { Azul }\end{array}$ & 1 & Madre, 3 hijos & & & no \\
\hline & & 14 & $\begin{array}{l}\text { Costa } \\
\text { Azul }\end{array}$ & 4 & Madre, 1 hijo (6 años) & & & no \\
\hline & & 15 & Centro & 4 & Madre, Padre, 1 hijo (6 años) & & & no \\
\hline & & 16 & $\begin{array}{l}\text { Costa } \\
\text { Azul }\end{array}$ & 1 & $\begin{array}{l}\text { Madre, } 3 \text { hijos (3 años, } 2 \text { y } \\
2 \text { meses) }\end{array}$ & & empleada & no \\
\hline & & 17 & Sur & 10 & $\begin{array}{l}\text { Madre, Padre, } 3 \text { hijos ( } 8,6 \\
\text { años, } 1 \text { mes) }\end{array}$ & 4o AP & & no \\
\hline 9 & Lucy & 18 & $\begin{array}{l}\text { Costa } \\
\text { Azul }\end{array}$ & 10 & $\begin{array}{l}\text { Madre, Padre, } 3 \text { hijos (12, } \\
\text { 10, } 4 \text { años) }\end{array}$ & $9 \mathrm{a} A \mathrm{P}$ & empleada & no \\
\hline \multirow{2}{*}{10} & \multirow{2}{*}{ Alfonso } & 19 & $\begin{array}{l}\text { Costa } \\
\text { Azul }\end{array}$ & 3 & $\begin{array}{l}\text { Madre, Padre, } 2 \text { hijos (6, } 1 \\
\text { año) }\end{array}$ & $10 \mathrm{AP}$ & niñera & sí \\
\hline & & 20 & $\begin{array}{l}\text { Costa } \\
\text { Azul }\end{array}$ & 10 & $\begin{array}{l}\text { Madre, Padre, } 2 \text { hijos }(8,5 \\
\text { años) }\end{array}$ & $10 \mathrm{AP}$ & abuelos & no \\
\hline \multirow{2}{*}{11} & \multirow{2}{*}{ Marina } & 21 & Centro & 1 & $\begin{array}{l}\text { Madre, Padre, } 3 \text { hijos (17, } \\
14,8 \text { años) }\end{array}$ & $2 \mathrm{a} A P$ & empleada & sì \\
\hline & & 22 & Centro & 11 & $\begin{array}{l}\text { Madre, Padre, } 2 \text { hijos }(6,6 \\
\text { años) }\end{array}$ & $2 \mathrm{a} A \mathrm{P}$ & empleada & no \\
\hline 12 & Eva & 18 & $\begin{array}{l}\text { Costa } \\
\text { Azul }\end{array}$ & 12 & $\begin{array}{l}\text { Madre, Padre, } 3 \text { hijos }(11,9, \\
3 \text { años })\end{array}$ & $8 \mathrm{a}$ AP & empleada & sí \\
\hline \multirow{2}{*}{13} & \multirow{2}{*}{ Guadalupe } & 23 & Norte & 5 & $\begin{array}{l}\text { Madre, Padre, } 3 \text { hijos }(11,4, \\
4 \text { años })\end{array}$ & $2 \mathrm{a} A P$ & & no \\
\hline & & 24 & $\begin{array}{l}\text { Costa } \\
\text { Azul }\end{array}$ & 4 & Madre, Padre, 1 hijo & $2 \mathrm{a} A P$ & abuelos & no \\
\hline 14 & Ana & 12 & $\begin{array}{l}\text { Costa } \\
\text { Azul }\end{array}$ & 9 & $\begin{array}{l}\text { Madre, Padre, } 4 \text { hijos }(20,5, \\
\text { 2, } 2 \text { años })\end{array}$ & 3a AP & & no \\
\hline
\end{tabular}




\section{Cuatro historias au pair}

Como hemos presentado al inicio, no existen normas que establezcan de manera clara cómo se desarrolla la experiencia au pair. Si tomamos en consideración los textos legislativos, hemos visto su ambigüedad y dejan abiertas múltiples opciones de interpretación de lo que significa la figura au pair. Para empezar a entender de qué diferentes maneras puede configurarse la experiencia de las au pairs, y qué variaciones puede asumir la relación entre ellos y las familias, empezaremos por proponer cuatro relatos que presentan una serie de temas y sus cambios según el contexto en el que se realizan.

\section{Dolores: "Parte de la familia, una hija más"}

La primera historia es la de Dolores, joven mexicana que llegó a la Costa Azul en el mes de agosto de 2015, después de haber contactado por internet a la familia ${ }^{15}$. Sus motivaciones para vivir la experiencia eran viajar fuera del país, conocer nuevos lugares y aprender otro idioma. Para ella ser au pair era lo ideal para realizar estos objetivos pues "me gusta estar sola, pero no vivir sola, me gusta estar con una familia porque alguien me puede ayudar". Su experiencia con los niños no había pasado del ámbito familiar: había cuidado a algunos niños como baby sitter y se había ocupado de sus sobrinos. Aunque llegó por contacto directo con la familia, tuvo también una agencia, aunque nunca les vio. A diferencia de muchos otros, no tuvo que pagarles nada, ellos solo sirvieron para realizar los trámites contractuales.

El padre de la familia tenía un cargo directivo en uno de los hospitales más grandes de la ciudad y la madre trabajaba en una clínica solo tres días por semana, por lo que estaba mucho en casa. El resultado es que Dolores estaba ocupada solo los días que la madre trabajaba, ocupándose de 3 niñas de 12, 6 y 3 años. Tenía que llevarlas a la escuela, después estaba libre hasta las $15 \mathrm{~h} 30$ cuando debía recoger a las pequeñas, les daba la merienda y a las $16 \mathrm{~h} 30$ recogía a la mayor; llegaban a casa y jugaban de 18 a 18h30, las duchaba y a las $19 \mathrm{~h}$ cenaban. Nunca tuvo que ocuparse de cocinar porque la madre era quien se encargaba; de $19 \mathrm{~h} 30$ a $20 \mathrm{~h} 30$ veían una película o hacían otras actividades, después ella les leía un cuento y las niñas se dormían. "Lo que me gusta de esta familia es que la mamá se ocupa mucho de las niñas, si ella llega yo estoy libre". En la familia, las tareas de cuidado eran en efecto prevalentemente femeninas, así que la referente principal de Dolores era substancialmente la madre.

Eran personas muy sociales, organizaban muchas comidas a veces con otros niños, pero esto a ella no le creó problemas, porque no le causaba demasiado trabajo. "Trabajamos en equipo la mamá y yo... Ellos están súper organizados, un mes antes ya saben todo lo que van a hacer". La relación con la familia marchó muy bien en general, se tuteaban y la hacían parte de muchas actividades. Las vacaciones de Navidad las pasó con ellos y conoció a las familias del padre y de la madre. Después se fue de vacaciones a Córcega y a esquiar con ellos. "Nunca tuve un problema con ellos. Jamás". Ella les define súper abiertos, y podía usar toda la casa: "soy parte de la familia, una hija más". Se consideraba muy afortunada con esta familia "tengo mucha suerte". La familia ha tenido au pairs desde hace 7 años: "no soy ni la primera

15 El sitio web más utilizado para convertirse en au pair o en familia de acogida es AUPAIRWORLD.COM. Cuando hablemos de búsquedas en internet, hacemos referencia a este sitio. 
ni la última". Ella cree que lo que ha funcionado es que han tenido mucha comunicación.

\section{Eva: "Esa no es mi familia"}

En los casos registrados, la mitad de las au pairs permaneció con la misma familia durante toda la estancia, lo que generalmente significó una relación exitosa. Sin embargo, no siempre la permanencia significaba estabilidad. Por ejemplo, tenemos la historia de Eva, una chica colombiana de 24 años. Las ganas de cambiar de rutina y de querer practicar el idioma fueron su motivación.

Eva llegó a Francia en septiembre del 2014, a una familia de 5 personas: padre, madre, dos niñas de 9 y 11 años y un niño de 2 años. El padre trabajaba fuera de la ciudad por periodos largos de tiempo, por lo que normalmente convivió con la madre y los niños. Esta familia había tenido au pairs durante toda la infancia de los niños. Sin embargo, cuando Eva llegó, la familia solo tenía una niñera temporal y no hubo quien le explicara las cosas, lo que le produjo cierto aislamiento que la mantuvo muy unida a la familia: "Cuando yo llegué eran dos meses que no tenían au pair, por lo que no hubo nadie que me explicó cómo iban las cosas, no me veía con nadie, no sabía nada, no sabía dónde estaban los pañales, fue como complicado. [...] Pasaba el $100 \%$ de mi tiempo con la familia, pero no lo consideraba trabajo. Al principio todo iba muy bien yo pasaba el tiempo con la madre y los hijos, pues el padre trabajaba de lunes a sábado y con él tenía el $0 \%$ de convivencia".

La rutina de Eva se desarrollaba con normalidad y ella, al pasar todo el tiempo con la familia, sentía un deber normal realizar diversas tareas; solo más adelante, cuando hubo problemas, su actitud frente a las tareas del hogar cambió totalmente y comenzó a verlas como responsabilidades que no le correspondía hacer.

Tuvo el primer choque con la familia durante una cena en la que llegaron unos invitados y, como no había lugar, le quitaron el puesto y la mandaron a una mesa aparte con el niño. "Ahí pensé, esa no es mi familia". En otra ocasión, tuvo un descanso de una semana y al regreso se dio cuenta que solo ella se ocupaba del niño. La actitud de Eva cambió: "Mis fines de semana me los pasaba en la habitación encerrada [...], sabía que si salía de mi habitación me tocaba trabajar. Jugar con los niños ya no me gustaba lo sentía como un trabajo". Su relación con el padre no mejoró: "El tiempo que pasaba con la madre y con los niños sin el padre era muy bonito. Nunca me llevé bien con el padre, era una relación mala. Cuando él me daba órdenes yo le contestaba mal. Me sentía muy sola porque todos mis amigos vivían lejos".

Tras las primeras discusiones, Eva buscaba tener más tiempo y espacio para ella, y sus actividades cambiaron: "Les pedí los fines de semana libres, pero ellos no estaban de acuerdo pues yo les había acostumbrado a estar siempre ahí". La delgada línea entre el trabajo como au pair y el tiempo libre "en familia" llega a ser un tema complicado; en el caso de Eva el enfrentamiento fue con el padre: "me decía 'tú no trabajas, eres parte de la familia". Para Eva fue difícil continuar con su estancia: "La situación se volvió hipertensa y yo empecé a hacer menos cosas. Al principio yo lavaba la ropa de ellos y hacía cosas de ménage pero después vi que si yo no lo hacía nadie lo hacía, entonces cosas que yo antes hacía por ser buena persona empecé a tomarlas como obligaciones y dejé de hacerlas. A ellos no les gustó". El contexto de esta familia reproducía roles de género marcadamente patriarcales, en los que la figura dominante era el padre. Por este motivo, la madre tenía un papel secundario 
en las discusiones y quien ejercía presiones e intentos de autoridad era el padre: "El padre siempre me amenazaba con correrme de la casa, cuando discutíamos él me decía 'vete de mi casa' pero otras veces ocurría al contrario 'tú tienes que ir con nosotros a la montaña eres de la familia'. El papá siempre me hacía sentir que era una trabajadora, aunque él decía que era parte de la familia".

En esta familia la casa era un departamento para 6 personas, el espacio jugaba un papel muy importante pues al parecer no había mucha intimidad. Incluso el hecho de compartir vacaciones contribuyó a aumentar la tensión: "Yo era la primera au pair que viajaba con ellos, pero para el papá lo mío era un trabajo y me daba órdenes todo el tiempo: lava los platos, cuida a los niños, etc.". Cuando su periodo como au pair estaba por terminar, los padres viajaron fuera del país durante todo el verano, dejándola sola a cargo de los niños. Muchos de las au pairs sienten que en ocasiones su trabajo no es sumamente necesario, sino que es sólo un espacio que los padres necesitan para estar sin sus hijos. "A mí me tocaba hacer cosas que corresponden a los papás, por ejemplo, yo le enseñé al niño a nadar. Normalmente existen unos nexos que se crean con los padres y él no tiene esos nexos. Yo le enseñé a ir al baño, a nadar, a ir al restaurante, aprendió a comportarse en la calle". El verano fue complicado: "fue horrible, yo fui mamá y papá al mismo tiempo, acudiente, no tenía vida. Pasó de todo: el niño se enfermó y la más grande se cayó en el colegio. La situación era angustiante". Al final Eva fue suplida por Lucy, otra colombiana. Actualmente estudia en la universidad y trabaja en algunos restaurantes. Sigue teniendo contacto con la familia.

\section{Alfonso: "Amigos cercanos, pero no mejores amigos"}

El caso de Alfonso, joven mexicano de 24 años, nos muestra cómo suele ser el cambio de familia. Alfonso decidió emprender la experiencia au pair para perfeccionar su francés e inscribirse en una maestría en Francia. Llegó a la Costa Azul a mediados de septiembre de 2015, pero su permanencia con la familia duró dos meses, y en noviembre cambió de familia. Con esta segunda familia la relación que se construyó fue mucho mejor, y se quedó con ellos 10 meses, hasta septiembre de 2016. Veamos de manera comparada la situación con la que se encontró Alfonso en las dos familias, intentando entender qué elementos jugaron un papel importante en determinar por una parte un fracaso, por otra un éxito de la inclusión familiar.

La primera familia a la que llegó Alfonso era de clase social modesta, el padre trabajaba como pastelero y la madre como profesora de hostelería. Ambos eran bastante jóvenes - unos 30 años - sin estudios de nivel universitario y con dos hijos, de 6 años y un año y medio. El apartamento donde vivían no era demasiado grande, y si bien Alfonso contaba con una habitación individual, el baño era compartido por toda la familia. Para ellos Alfonso era el primer au pair, y lo necesitaban a causa de las condiciones y horarios de trabajo: el padre trabajaba de 8 am a 8 pm y la madre tenía que trasladarse fuera de la ciudad durante la semana. Ambos provenían del norte de Francia, y no tenían familia cercana que pudiese ayudarles. Por eso, su interés en acoger a Alfonso en su casa era más por necesidad que no por el deseo de embarcarse en una experiencia de intercambio cultural. En cuanto a las tareas Alfonso dice: "me tocaba hacerme cargo de los niños todo el día. Los tenía que levantar, vestirlos, darles de desayunar, llevarles a la escuela. Recogía el cuarto de los niños y también me ocupaba de lavar su ropa, por la noche los duchaba, cocinaba, les daba de cenar y los llevaba a la cama con un cuento". 
Desde el principio entre Alfonso y la familia no se consiguió crear una situación de "familiaridad", pues a pesar de la joven edad de los padres, el trato entre ellos fue muy formal: "el padre era serio pero al principio se mostró muy paciente. Yo le hablaba de usted, y él nunca dijo nada al respecto. Con la madre también existió esta situación". En este marco de distancia formal, la relación empezó a sembrarse de pequeños desencuentros. El primero de ellos fue relativo al auto: Alfonso tenía que usarlo para llevar al niño pequeño a la niñera, "sin embargo, cuando la madre me mostró el camino y vio cómo conducía se molestó, dijo que le había mentido y que no sabía conducir". Los desencuentros continuaron en otros ámbitos de la vida cotidiana, pues la madre era muy exigente y Alfonso a veces no comprendía bien las instrucciones: "nunca se caracterizó por ser paciente". La convivencia cotidiana se volvió caracterizada por reproches continuos: "Un día que usé la televisión el padre se molestó porque no la había dejado en el mismo canal. Una vez comí una manzana que había en casa y la madre me reclamó que ella tenía pensado usarla para cocinar". La falta de resolución de estos problemas hizo que la relación se volviese en poco tiempo extremamente distante, al punto que el padre dejó de hablar a Alfonso: "fue dejando el trato en la cortesía, sólo nos decíamos bonjour y luego ni eso, después yo lo saludaba y el solo respondía con un gesto moviendo la cabeza". Estas tensiones influenciaron también la relación de Alfonso con los niños: "Para mí fue difícil estar con los niños porque el pequeño lloraba casi todo el tiempo y el grande nunca quería hacerme caso porque decía que no me entendía". Para Alfonso las cosas no iban bien: "la situación no era del todo agradable para mí, no me sentía a gusto, vivía siempre estresado", y "cada vez que podía, salía de la casa lo máximo posible ya que ahí me sentía como enjaulado, vigilado, presionado". Las salidas de Alfonso, en lugar de facilitar la convivencia — pues aparentemente habrían podido ayudar a que se encontraran espacios de autonomía - crearon aún más tensiones.

Ante las dificultades, "la madre llamó un par de veces a la agencia y ellos pidieron hablar conmigo. Yo sentí que en estas entrevistas me decían que tenía que esforzarme más, no me sentí apoyado sino exigido a responder bien. Cuando le dije a la agencia que quería cambiarme de familia, me dijeron que no había opciones, era quedarme ahí y hacer el esfuerzo o quedarme sin nada". Frente a la aparente imposibilidad de resolver la situación, Alfonso decidió buscar otra familia por su cuenta, y otras au pairs que tenían ya experiencia de cambio le asesoraron sobre los trámites, tranquilizándole sobre todo sobre su situación migratoria. Fue muy importante, pues en los discursos de las agencias siempre está presente de forma más o menos explícita la amenaza de repatriar a la au pair que falte a sus deberes ${ }^{16}$.

A mediados de octubre Alfonso encontró otra familia y en noviembre se mudó con ellos. Esta familia era bastante diferente, comenzando por las edades y el nivel social de los padres. El padre tenía 50 años y la madre 42, y ambos trabajaban en el sector financiero. Tenían dos hijos de 9 y 5 años, y vivían en una villa muy grande, con jardín y piscina. Alfonso disponía de un mini apartamento con baño propio, cocina y entrada individual. Las condiciones de convivencia eran del todo diferentes: "Tenía mucha privacidad ahí, era como mi casa porque el espacio era sólo mío". La situación mejoró y muy rápidamente se instauró con la familia una relación muy buena. También para esta familia Alfonso era el primer au pair, y le acogieron más

16 La vulnerabilidad laboral que deriva de la condición migratoria de las au pairs y de las trabajadoras domésticas en general ha sido evidenciada en el caso británico por Anderson (2007). 
por necesidad que por deseo de establecer un intercambio cultural. El trato continuó siendo formal -hablándose siempre de usted - y las ocupaciones eran similares: "Los niños salían a las 16h de la escuela. Cenaba con la familia y a eso de las 8 o 9 ya podía bajar a mi apartamento. Recogía el cuarto de los niños, lavaba su ropa, recogía un poco la cocina las mañanas y una vez por semana hacía la limpieza de la sala de estar y del cuarto de los niños. Los fines de semana era libre". Igualmente, parecidos fueron los recelos relativos a la privacidad y a la convivencia: "el padre me dijo que temía que esta situación de compartir la intimidad con alguien más le iba precisamente a dificultar un poco la posibilidad de descansar al 100\%, de 'sentirse en su casa', pero [al final de la estancia] me dijo que apreció que yo fuera muy discreto y que haya comprendido casi de manera natural haber dado su espacio familiar."

Lo que cambió fueron las condiciones de contexto que facilitaron la convivencia cotidiana - espacios más grandes, posibilidades de aislamiento - pero también la capacidad de Alfonso y de los padres de reaccionar a las dificultades, construyendo un equilibrio entre inclusión y autonomía. Alfonso pasaba la mayor parte de su tiempo libre fuera de casa sin que esto constituyera un motivo de reproche, pero también fue incluido en actividades de tipo familiar: conoció y tuvo buen trato con los abuelos maternos, acompañó algunas veces a la familia al golf y "compartí con ellos algunos momentos de loisir como la final de la Eurocopa o las vacaciones de febrero, donde me llevaron con ellos a Suiza".

El resultado fue una experiencia positiva, si bien no se creó nunca una inclusión familiar efectiva: "Llegué a sentirme muy cómodo con ellos, siempre hubo una relación de respeto, era claro que no era parte de la familia y que mi estancia ahí no dejaba de ser un trabajo, pero se forjó una relación que yo diría como de amigos cercanos, pero no de mejores amigos".

\section{Marina: "un miembro de la familia no, pero sí una persona"}

Otra historia de cambio de familia de una situación negativa a otra positiva le sucedió a Marina, mujer colombiana de 24 años. Llegó en septiembre de 2015 a un pequeño pueblo del centro de Francia, a una familia con 3 niños de 17, 14 y 8 años. La motivación para ella era también la posibilidad de estudiar en Francia ya que no estaba contenta con su trabajo en Colombia. Desde un principio la situación fue un tanto incómoda: llegó a París y la familia no estuvo allá para recibirla; después de una espera de tres horas la familia envió un taxi para ir a buscarla; una vez ya en casa el recibimiento fue frío.

Otro de los problemas de adaptación de Marina fue la falta de privacidad, pues su cuarto servía también como almacén, y la familia entraba a tomar o dejar cosas cuando quería, sin pedir permiso. La apropiación de espacios como propios juega un rol muy importante en la experiencia de las au pairs (Búriková, 2006), ya que la habitación muchas veces llega a ser un símbolo de apropiación, un refugio, o, por el contrario, un lugar en el que no se quiere dejar huella. La falta de privacidad puede ser chocante para las au pairs, pues les puede hacer sentir vulnerables, dado que su persona no es respetada sino ignorada en cuanto a "tiempo a solas" o "espacio personal" se refiere.

La ubicación de la población hizo sentir a Marina aislada: era un pueblo pequeño a 40 minutos en auto de la ciudad más grande. Mucho del éxito o fracaso de la relación au pair-familia depende también, en cierta medida, de la posibilidad que 
tengan para socializar o realizar otras actividades fuera del ámbito familiar. Es por este motivo que si el lugar es muy pequeño al final no complace las expectativas de la au pair, y se puede volver un factor importante en el momento de permanecer o no con la familia ${ }^{17}$. Marina, los fines de semana, se veía obligada a quedarse en casa y la familia comenzó a dejarle más tareas, sobre todo el cuidado de la niña más pequeña, mientras ellos salían. Después de dos meses, la situación se volvió intolerable hasta tal punto que comenzó a tener repercusiones en su salud: se sentía mareada, débil y tenía vómitos. Comunicó esto a la familia, pero ellos nunca la llevaron al médico.

Marina había viajado a Francia por medio de una agencia, y pese a comunicar siempre sus problemas y sus deseos de cambiar de familia, ella sentía que la agencia no hacía mucho: "la chica me escuchaba y decía que me iban a mover, pero yo sentía que no se movía". Al igual que en otros casos, las agencias tienden a mostrarse ausentes: si las cosas van bien y no hay ningún problema nunca se hacen presentes, si las cosas van mal y las au pairs piden ayuda, la agencia parece no hacer uso de muchas de sus herramientas. Alfonso comentaba que cuando quiso cambiar de familia la agencia no le daba muchas alternativas y le presentaban la situación como fija e inmodificable. Eva nunca supo de su agencia, y ni siquiera le vino la idea de llamarla cuando tuvo problemas.

Marina decidió al final buscar otra familia e irse, pero el día que se lo comunicó al padre, este se molestó y le reclamó una serie de gastos e incluso la insultó. Ella se desestabilizó y llamó a una ambulancia, pero el padre no dejó que la atendieran de inmediato. Al final, tuvo que intervenir la policía y ella fue llevada a un refugio donde pasó varios días hasta que una de sus amigas la pudo ir a buscar.

Después de este episodio, Marina logró encontrar otra familia en la ciudad, donde parece que desde un principio las cosas marcharon bien: "Nos encontramos muy bien. Ellos tuvieron conmigo una paciencia enorme. Fueron muy lindos conmigo. Yo estaba muy triste por todo lo que había pasado, y ellos estaban muy pendientes de mí, la madre venía siempre a hablarme. Me sacaron de la depresión”. Marina tenía aquí más privacidad en su alcoba y tenía incluso baño propio. Había una femme de ménage, por lo que ella se dedicaba exclusivamente a los niños y logró continuar con el estudio del francés. La familia era inclusiva y la hacía sentir "parte de" al compartir ciertos habitus: "los viernes eran de pizza y ellos pedían siempre para mí". Le presentaron a la familia y también a sus amistades más cercanas, "conocí sólo personas bonitas". Era la tercera au pair en esta familia y los padres tenían muy clara una idea de inclusión y de descubrimiento del otro: "les gustaba hacerme descubrir cosas francesas, y ellos descubrir cosas de Colombia, yo les cocinaba muchas cosas... me mostraron varios platillos típicos de la Francia y se ponían contentos cuando yo les cocinaba algo colombiano".

Muchas dificultades derivan del hecho de que las jóvenes se enfrentan por primera vez a esta situación; por tanto, el qué hacer y cómo actuar no es del todo claro, sobre todo si se toma en cuenta que las acciones se desarrollan en otro país, lo que implica que el código de comportamiento sea forzosamente diferente y por tanto no muy fácil de seguir al principio. Marina terminó su estancia como au pair en esta familia y se quedó en Francia con la esperanza de encontrar un trabajo. Pese a que su salud se vio afectada por la situación de la primera familia, ella se va satisfecha

17 Por lo menos otros dos au pairs - Pablo y Paulina - tomaron la decisión de marcharse de esa familia debido a las reducidas posibilidades de realizar actividades fuera de casa, ya que la población era realmente pequeña. 
de la relación que entabló con la segunda familia: "Tal vez un miembro de la familia no, pero sí una persona".

\section{La construcción de la relación entre au pairs y familias}

A partir de estos relatos, vemos cómo la experiencia de las au pair sucede de maneras muy diferentes. Estos relatos no pretenden ser ni exhaustivos ni ideal-típicos, sino sólo explorar las variaciones que puede presentar la dinámica entre au pairs y las familias. La vaguedad legislativo-normativa de la institución au pair la convierte en una especie de marco que contiene múltiples posibilidades de realización. Como han escrito Búriková y Miller (2010), el sistema au pair es una institución altamente informal que deja espacios a la discreción de su interpretación. Alargando el horizonte no solo a estos cuatro relatos, sino a todos los 14 casos, intentaremos ver ahora cuales son los elementos que pueden determinar los diferentes tipos de experiencia.

\section{Habitus y culturas de familia}

En primer lugar, encontramos la cuestión de la "familia". Las y los au pair, supuestamente, tienen que integrarse en una familia, pero de qué familia estamos hablando no es claro. El avance de la segunda transición demográfica en Francia ha impulsado la fragmentación de las formas de familia, creando un panorama que Bob Simpson (1994) ha definido como el paso de la nuclear family a la unclear family. Este proceso de "unclearización" hace difícil definir formas y estructuras. En los casos que hemos estudiado, por lo menos tres familias eran recompuestas, con la convivencia - permanente o periódica- de hijos de matrimonios anteriores de los padres. No siempre los padres de las familias estaban casados, sino que, en varios casos, simplemente convivían. Nos hemos encontrado también con familias monoparentales, y con familias donde por motivos de trabajo, el padre o la madre pasaban parte de la semana fuera de casa. Muchas de las familias estaban formadas por matrimonios jóvenes, pero otras respondían a la definición de "familias tardías", en la que los hijos llegan a una edad avanzada de los padres. En algunos casos estaban presentes fuertes redes de parentesco alrededor de la familia, y las y los au pair convivían casi cotidianamente con figuras como abuelos, tíos y primos de los niños, pero en otras el núcleo estaba alejado de cualquier contacto frecuente con sus parientes.

Esta fragmentariedad familiar nos empuja a concentrarnos en la manera en que cada persona concibe e interpreta las situaciones familiares, es decir sobre el concepto de "culturas del parentesco" (Piasere y Solinas, 1998), sobre los habitus de relacionalidad (Carsten, 2000, 2004) que se construyen en los espacios domésticos (Grilli, 2008). La superposición de formas familiares tan diferentes supone una redefinición de los valores y significados atribuidos a las relaciones de familia y parentesco, y siguiendo algunos hilos conductores podemos intentar explorar este complicado panorama.

Siguiendo la pista de las culturas de familia, podemos ver cómo la situación familiar donde se encuentran las au pair es un escenario de confrontación, en el que entran en contacto culturas y habitus de familia diferentes entre ellos: por una parte, los de la familia que acoge - los padres y los hijos-, por otra, la de las au pair. Esta confrontación constituye un proceso de negociación y construcción de una re- 
lación que no puede ser definida a priori por normas y reglas, sino que tiene que ser probada y experimentada en la vida cotidiana, a través de la creación de un espacio doméstico de convivencia común (Cox y Narula, 2003). Esta visión procesual de la relación entre au pair y familias nos lleva a relativizar y considerar de manera diferente varios elementos. Para orientarnos haremos ahora un recorrido transversal de todos los casos, en el que reflexionaremos sobre qué elementos pueden tener una función de orientación en la construcción de la relación entre au pairs y familias.

\section{Las intenciones}

El primer elemento que influye mucho en el proceso y que diferencia las situaciones son las intenciones y expectativas de las familias y las y los au pairs respecto a la relación que hay que establecer. No todos tienen, en efecto, la intención de empezar este proceso de negociación e inclusión familiar. Existen familias que quieren tener una au pair como experiencia cultural, y muchas otras cuya acogida está determinada por necesidades de ayuda y cuidados, que no se pueden satisfacer de otras maneras. Al mismo tiempo, entre las y los au pairs están quienes desean tener una experiencia de intercambio cultural, pero también los que utilizan esta actividad como una estrategia migratoria para llegar a Europa de manera simple y sin muchos trámites. El cruce de las variables nos crea cuatro tipos de potenciales situaciones ideales:

a) La familia y la/el au pair tienen expectativas mutuas de relacionalidad. En esta situación, ambos se ven potencialmente como miembros de una familia común.

b) La familia tiene expectativas de intercambio, mientras que la/el au pair ve su experiencia más como una estrategia migratoria. En esta situación, la familia está dispuesta a integrar a la au pair, mientras esta ve sus funciones más "como de trabajo".

c) La familia tiene más necesidad de ayuda y cuidado que expectativas de relacionalidad, mientras que la/el au pair se espera una experiencia de intercambio cultural. En esta situación, la familia considera a la au pair como una trabajadora, mientras que esta estaría dispuesta a establecer lazos más fuertes en la dirección de la parentalización.

d) La familia y la/el au pair tienen motivaciones utilitarias: la familia necesita ayuda, la/el au pair quiere llegar a Europa. En esta situación, ambos consideran que la relación es de trabajo y nada más.

Estas situaciones no deben ser consideradas de manera rígida y separada, pues pueden presentarse de manera matizada y con intensidades variables, además de que las personas pueden cambiar sus motivaciones $\mathrm{y}$, por tanto, las situaciones pueden transformarse. Hemos visto, por ejemplo, como muchas au pair llegan con intenciones de intercambio, pero encontrándose en una situación de tipo (c) se desilusionan y su motivación cambia hacía una situación (d), como en el caso de Eva. En otros casos, los malentendidos pueden llevar a transformar la situación como en el caso de Alfonso, donde la falta de entendimiento con la primera familia hace que una situación de tipo (a) se convierta casi enseguida en (c), y de ahí vaya a la ruptura.

Si podemos pensar que la situación (a) es la más favorable para un proceso que conduzca a una parentalización, esto no es automático, y la falta de compatibilidad entre habitus y culturas de familia puede llevar en cambio al fracaso de la relación o a otras situaciones. Uno de los ejemplos de esta falta de compatibilidad está conectado al sentido y valor que las y los au pair dan a su propia presencia, si se consideran 
útiles o no. Muchas au pair llegan con la idea de que la creación de una solidaridad familiar tiene sentido solo si su presencia es necesaria, y en la medida en que consideran útil la tarea de cuidado están dispuestas a hacerlo. De lo contrario, consideran que su presencia es fruto de una especie de capricho que roza la explotación laboral.

Por ejemplo, Margarita consideraba que la madre de la familia en realidad no la necesitaba, pues tenía mucho tiempo libre que habría podido dedicar a sus hijos. Marta consideraba que la madre trabajaba solo por gusto, y que no habría sido necesario gracias al hecho de que el padre percibía un buen salario. Por tanto, el no poder ocuparse de los hijos no era visto por ella como algo positivo. Eva evaluaba muy negativamente el hecho de que la madre a veces prefiriese crearse espacios de recreación, como tomar café con las amigas, en vez de pasar el tiempo con sus hijos. En todos estos casos, vemos una diferencia de concepción de la idea de familia, en la que las au pairs no comparten las ideas de emancipación y autonomía de las madres de clase media-alta europea. A pesar de las intenciones originales, esta diferencia constituía un obstáculo para la realización del proceso de parentalización, y efectivamente en todos estos casos la relación se transformaba en una relación más formal de trabajo doméstico.

\section{Habitus de familia y flexibilidad}

En el ámbito del proceso, la capacidad de superar los malentendidos y de negociar entre respectivos habitus familiares juega un papel muy importante. Aquí vemos que familias y au pair pueden reaccionar de manera diferente. Las y los au pairs tienen la particularidad de encontrarse en una fase de la vida que Búriková y Miller (2010), siguiendo el esquema de los ritos de paso, han propuesto definir como "liminal". El hecho de que para la mayoría de las au pair la experiencia sea no solo la primera salida de casa, sino que también muchas veces sea la primera salida de su país, y el hecho además de que sepan que es una situación temporal, justifica la pertinencia de aplicar la categoría de liminalidad a su situación. La particularidad de la fase liminal es la de ser un momento de suspensión de las normas sociales, en la que los sujetos buscan y experimentan opciones y experiencias antes de reincorporarse a la sociedad. Las maneras en las que se vive esta fase pueden ser muchas: por una parte, puede efectivamente haber un deseo de experimentación y novedad que les hace más adaptables; por otra, la lejanía de casa y la soledad les puede llevar a cerrarse y a poner en acto mecanismos de reificación y reafirmación de elementos de las culturas de origen.

Reacciones muy diferentes a los conflictos pueden ser determinadas también por las intenciones que motivaron su experiencia. Si la experiencia au pair se debe a estrategias migratorias, es más probable que la/el au pair presente menos disposición a negociar, y que, de manera parecida a lo que pasa con muchos grupos de migrantes, se encierre en una reafirmación de su legado cultural de origen. Esta actitud se puede apreciar a través del modo en que las y los au pairs configuran su red de amistades: por ejemplo, en casos como los de Dolores, Ricardo y Ana, cuyas motivaciones de viaje eran expresamente de intercambio cultural, el círculo de amistades del que se rodeaban era bastante variado, con la presencia de franceses o de no latinoamericanos. En otros casos, en cambio, como los de Eva, Pamela, Alejandra, Lucy y Dolly, cuyas motivaciones parecen ser más de tipo migratorio, la red de amistades estaba formada en su mayoría por otras au pair de su misma nacionalidad. En efecto, en 
los primeros casos encontramos situaciones de inclusión familiar más conseguidas, mientras que en los segundos la relación o está llena de desencuentros, o se configura desde el principio como una relación de trabajo.

También entre las familias encontramos disposiciones diferentes hacia las diferencias, que van del querer imponer sus habitus sin negociar — como en el caso de la primera familia de Alfonso - a tener efectiva curiosidad y aceptar las novedades, como en el caso de Ricardo y Ana, o de Marta y Dolores. La segunda familia de Marina favorecía particularmente intercambios en el ámbito de la cotidianidad, a través, por ejemplo, de intercambios de recetas. Otras familias, en cambio, o no parecen estar interesadas, o son explícitamente más cerradas, como en el caso de Alejandra. En una ocasión en la que ella intentó negociar cuestiones de convivencia — no hacer ruido por la mañana temprano el fin de semana - la madre le contestó con un SMS "muy largo y grosero, cuyo sentido era esta es mi casa y no me jodas. Tuve que aguantarme y no dije nada".

A este respecto, el número de au pairs que tiene una familia puede ser índice de actitudes diferentes. En algunos casos, el tener muchos au pairs es efectivamente fruto de un interés por la experiencia y por querer empezar procesos de relacionalidad. Este es el caso de Ana y Ricardo, respectivamente tercera y cuarto au pair de una familia muy dispuesta a integrarles. En otros casos, el tener muchas au pairs en cambio es casi una mecánica, que anula cualquier esfuerzo de adaptabilidad por parte de la familia. La familia de Margarita había tenido antes de ella otras 11 au pairs y, según su relato, le imponía un estricto protocolo ya experimentado, dejando pocos espacios a la construcción de una relación diferente de la que ellos querían. En el caso de Eva y Lucy, respectivamente octava y novena au pair de la familia, esto se reflejaba en un juego de continuas comparaciones con las precedentes que, en su impresión, limitaba su esfera de acción y negociación. Dolly estuvo con una familia solo un mes de verano, siendo la au pair número once de una niña de 8 años. En este caso es evidente cómo la familia, más que querer tener una relación de intercambio cultural, necesitaba a una niñera de verano a bajo coste. En efecto, Dolly no tuvo posibilidad de instaurar una relación con la familia y tuvo que adaptarse a todo lo que le decía la madre. La falta de interés de la familia llegaba al punto que la empujaban a salir de casa lo más posible en sus momentos libres, para poder pasar tiempo a solas entre ellos. Dolly no consiguió encariñarse tampoco con la niña, ya que esta estaba ya acostumbrada al cambio de au pairs y la consideraba una persona de paso en su vida.

\section{Construir la vida en común: tareas, horarios y espacios}

Otros tres elementos que hemos visto en los relatos juegan un papel fundamental en la construcción de la relación entre au pairs y familias: las tareas, los horarios de la vida doméstica y los espacios donde esta se desarrolla. La visión procesual nos ayuda a ver de manera diferente todos estos elementos, y sobre todo la importancia que tienen en determinar la forma que va a asumir la construcción de la relación.

Empezamos por las tareas, es decir qué tareas son propias de la au pair y cuáles no, qué tareas constituyen una actividad efectivamente familiar y cuáles, en cambio, ya podrían ser consideradas servicio doméstico. Los resultados de nuestra investigación nos llevan a pensar que la definición de las tareas es un falso problema. Lo que cuenta no es la tarea en sí, sino la percepción que se tiene de ella, así como el modo a través del cual la tarea viene asignada por la familia y recibida por la au pair. 
Hemos visto cómo tareas similares pueden ser interpretadas de manera diferente según la situación: arreglar las habitaciones de los niños o cocinar para ellos es visto por algunos como una ayuda normal, mientras que otros lo consideran como algo no apropiado a su situación. Es frecuente que se interprete de manera diferente la misma tarea en fases sucesivas. Eva, al principio de su permanencia, pasaba todo el tiempo con la familia, "pero no lo consideraba trabajo", mientras que al final, hasta el hecho de jugar con los niños "lo sentía como un trabajo". Margarita, al principio, lavaba la ropa de la familia "por reciprocidad", pero cuando terminó haciéndolo solo ella ya empezó a sentirlo como una forma de explotación. Alejandra, al principio hacía tareas como limpiar toda la casa y cocinar para toda la familia "porque me nacía hacerlo" y "porque estaba acostumbrada a que en mi casa en Colombia era igual", pero cuando notó que la familia se había acostumbrado a sus favores y ya los daba por normales, se molestó y dejó de hacerlos. Más que reflexionar sobre las listas de tareas, puede ser por tanto útil reflexionar sobre la manera en que estas son asignadas y percibidas por las y los au pairs ${ }^{18}$, pues es su percepción más que su contenido lo que hace que el proceso de negociación de la relación tome un camino u otro.

Las tareas están genéricamente conectadas a un horario, por lo que las maneras de entender las primeras se reflejan también en la percepción que se tiene del segundo. Sin embargo, la informalidad, a veces, impide un registro "imparcial" de las horas de actividad según la percepción tanto de las y los au pairs como de la familia. Dolly nos mencionó que una de las causas de fricción entre ella y la familia fue que le hacían trabajar más horas de lo previsto, encargándole tareas de limpieza que le costaban 3 horas extra, o querían que estuviera siempre a disposición por si necesitaban de ella. Caso contrario era el de las familias de Pamela, Alfonso o Dolores, donde las horas trabajadas en la semana no llegaban a las 30, por lo que, en las situaciones en que las familias necesitaban su presencia, ellos se mostraban flexibles pues sentían tener que cumplir como "un deber moral". Examinar la "economía moral" interna en el grupo doméstico nos remite a la perspectiva de Marshall Sahlins (1965), el cual, significativamente, utiliza justo el concepto de "reciprocidad" (mutuality) - reportado anteriormente por Margarita- como elemento característico de las transacciones económicas y laborales internas al grupo familiar. En la perspectiva de Sahlins, elaborada también en fases sucesivas (2013) y aplicada también al contexto francés por Attias-Donfut, Lapierre y Segalen (2002), es la reciprocidad el principio que construye el grupo doméstico y el parentesco. En el ámbito de la familia, el reparto de tareas y las transacciones de tipo económico-laboral se caracterizan por una lógica de intercambio que niega lo "económico" (1965). Dado que según Sahlins el rango o la distancia parental se miden según la presencia gradual de la reciprocidad en los intercambios, por nuestra parte podríamos sugerir que la distancia entre las ideas de parentalización y relación laboral entre las y los au pairs se juega de manera importante sobre la percepción de las tareas en cuanto reciprocidad familiar o intercambio económico-laboral.

Aunque por razones de síntesis no podamos profundizar el tema, señalamos que también la gestión de los espacios juega un rol muy importante en la construcción de

18 Gran parte de los estudios sobre las au pairs se han concentrado mucho sobre los tipos de tareas, llegando a hacer listas (Cuartas Villa, 2014) en los que se intenta discriminar entre tareas pertinentes a la familia y tareas más propias del servicio doméstico. Búriková y Miller (2010) llegan a pensar que una reglamentación de las tareas sea el primer paso para definir mejor el rol de las au pair y eventualmente tutelarlas de abusos. 
la relación (Eleb, 1996), y está profundamente conectada a las tareas y a los horarios. Normalmente las y los au pairs tienen un espacio autónomo, pero esta autonomía depende de las intenciones de la familia y tiene un impacto sobre la relación. Por ejemplo, Margarita, en el caso de la primera familia, tenía una habitación que estaba colocada en medio de las de los niños que cuidaba, y a ella le parecía que, más que un gesto de inclusión, era una estrategia para tenerla siempre pendiente y ocupada de ellos. Ya hemos visto cómo en muchos otros casos las "au pair" no sentían tener privacidad, pues sus espacios eran utilizados también por la familia. Es el caso de Marina, Eva y Lucy, cuyas habitaciones eran usadas para guardar cosas de la familia, por lo que siempre había algún motivo para que alguien entrara sin que ellas lo esperaran. Dolores, Marta, Alfonso y Pamela contaban en cambio con espacios separados de la casa, y esto permitía que ambas partes tuviesen sus propios espacios de intimidad.

\section{Desigualdades}

Como último punto, es importante señalar que, si bien hemos pasado en reseña qué elementos pueden ayudar a orientar la construcción de la relación entre au pairs y familias, no tenemos que olvidar que todo esto se realiza en un escenario profundamente marcado por desigualdades de género, clase y raza. Estas desigualdades nacen desde los mismos contextos familiares, pues a pesar de que en algunos casos se presenta una situación de roles familiares bastante equitativa, buena parte de las familias reproducía esquemas patriarcales de desigualdad y jerarquía de género típicos del mundo mediterráneo, en los que, si bien las labores domésticas eran asignadas a la madre, las decisiones importantes sobre el hogar eran asumidas por el padre. Estos esquemas marcan a veces desde el principio la relación entre familias y au pairs, sea que esta se oriente hacia una parentalización, o hacia una subordinación laboral de las au pairs. En efecto, el proceso de parentalización puede asumir las formas de integración dentro de un sistema de parentesco patriarcal y machista, donde a las mujeres corresponde un papel subordinado respecto al hombre. Encontramos esta desigualdad en la asimetría en el reparto de tareas, por lo que en muchos casos las au pairs se encuentran realizando sus labores casi exclusivamente con las madres, o se encuentran viviendo situaciones donde el padre reproduce con ellas la asimetría de género de la familia. Igualmente, hemos podido ver cómo la condición migratoria de las au pairs las convierte en sujetos extremamente vulnerables, cuya capacidad de acción es limitada por el temor a que las familias rompan unilateralmente la relación y las expongan a situaciones de irregularidad migratoria (Anderson, 2007).

\section{Conclusión: las contradicciones au pair, entre la construcción de un parentesco ideal y un servicio doméstico encubierto}

Las ideas que nos han guiado en nuestra exploración del universo au pair son dos. En primer lugar, hemos querido reflexionar sobre cómo las relaciones de cuidado y asistencia familiares pueden ser ámbitos favorables para la construcción de lazos asimilables al parentesco ficticio. En segundo lugar, sabiendo que la figura au pair viene presentada de manera oficial como una manera de incluirse e integrarse en una familia, hemos querido investigar las maneras en las que esta eventualidad llega a realizarse o no, en este último caso originando relaciones que son más asimilables al trabajo y al servicio doméstico. 
Lo que hemos llegado a ver es cómo la institución au pair, gracias a su ambigüedad y a su poca normatividad de base, se presta a una variedad de posibilidades, según toda una serie de componentes muy diferenciados que hemos visto cruzarse y sobreponerse entre ellos. La institución au pair puede verse como una especie de contenedor vacío revestido de discursos metafóricos de inclusión familiar y parentalización. La cuestión es que estas metáforas de relacionalidad y parentalización no tienen de por sí ningún efecto, pues como señala Janet Carsten (2004), para realizarse necesitan normalmente tiempo, constancia y aplicación por parte de las personas implicadas. En nuestro caso, además, las normas efectivas de esta institución social son extremamente vagas y no contienen dispositivos que pretendan actuar en los discursos que las revisten. La figura au pair se funda sobre una idea algo improvisada, que es la de una familia nuclear en la que nuevos miembros pueden integrarse en la medida en la que conviven y ayudan en el cuidado de los hijos. El estatus de familiar es pues conectado explícitamente a las funciones de cuidado de los hijos, así como a la convivencia bajo un mismo techo.

El problema es que el sistema au pair no es una institución consolidada de parentesco ficticio como otras, pero esto no quiere decir que no tenga la potencialidad para serlo, y que en algunos casos pueda llegar a realizarse de esta manera. El hecho de que la relación entre au pairs y familias se base sobre el cuidado y la asistencia, la hace potencialmente favorable para la creación de procesos de parentalización, según la idea de que en la sociedad contemporánea sur-europea no es tanto el parentesco el que produce cuidado, sino más bien es el cuidado el que produce parentesco (Zanotelli 2010).

Pero, ¿por qué esta potencialidad no siempre se realiza? En nuestra investigación hemos intentado explorar los factores a través de los que la relación entre familias y au pairs puede tomar caminos diferentes: algunos van en la dirección de la creación de parentesco ficticio, otros en cambio configuran la relación como una forma de prestación laboral encubierta. Estos factores pueden ser diversos y pueden combinarse de manera diferente en el curso del proceso de construcción de la relación. Este proceso no es simple ni breve ${ }^{19}$, y no podemos negar que en la mayoría de los casos responde a motivaciones utilitarias, tanto por parte de las familias como de las y los au pair. Muchas familias ven en la figura au pair la posibilidad de obtener una prestación de cuidado profesional poco regulada y a bajo coste, mientras que muchos jóvenes latinoamericanos ven la experiencia como una estrategia migratoria para llegar más fácilmente a Europa.

Es, pues, correcto hablar de las "múltiples facetas del au pair" (Cuartas Villa, 2014), en el sentido que la institución tiene en sí la plasticidad para configurarse de maneras muy diferentes, que en la mayoría de los casos no responden al nivel discursivo que la define. Este nivel discursivo se funda sobre el presupuesto político-ideológico que considera a la familia como la institución social a la que corresponden las principales funciones de cuidado, asistencia y solidaridad social de sus miembros (Pitrou, 1996; Carsten, 2004). El hecho de que la familia nuclear, con la extensión

19 Por razones de espacio no conseguimos tratar en el marco de este artículo un punto muy importante, o sea la temporalidad necesaria para la realización del proceso de parentalización. La retórica del programa au pair parece olvidar que los lazos relacionales necesitan tiempo para construirse, y que no es posible activarlos automáticamente solo con base en definiciones de la situación. Al mismo tiempo, el hecho de que ambas partes — familias y au pairs — sepan que su relación tiene un término — un año, máximo dos — influencia seguramente las direcciones del proceso y sus resultados de una manera que sería interesante profundizar en futuros estudios. 
eventual de los abuelos, sea en las sociedades mediterráneas la institución principal demandada para el cuidado se ha vuelto un debate al mismo tiempo académico y político (Martin, 1996), que los procesos socio-demográficos de desparentalización y la reducción progresiva del Estado del bienestar han alimentado.

La figura au pair es parte de esta idealización política y social de la familia en el ámbito del cuidado, pero paradójicamente no contiene en sí los dispositivos para configurarse automáticamente en esta dirección. La figura au pair es en efecto una institución relativamente nueva en el panorama sur-europeo, no pertenece al repertorio de formas sociales y culturales que definen la asistencia y el cuidado familiar. Igualmente, a pesar de las posibles similitudes con el panorama familiar europeo - la idea de "lazos fuertes" - tampoco en el contexto latinoamericano existen instituciones sociales de cuidado parecidas. Por tanto, es una institución social nueva y lo que evidencia nuestra investigación es que es extremamente improvisada. En todos los casos que hemos examinado, familias y au pairs tienen ideas y motivaciones diferentes sobre cómo vivir la experiencia, e improvisan su actuación. Por esto, nos encontramos delante de procesos de construcción de relaciones bastante imprevisibles, en los que, justo porque no existen reglas, cada mínimo factor puede tener una importancia decisiva en orientar el resultado.

La institución au pair puede ser vista, por tanto, como un espacio de experimentación social, en el que emergen todas las contradicciones y asimetrías del modelo de asistencia familista mediterráneo, suspendido entre la necesidad de asistencia y cuidado, la falta de parientes y la falta de recursos para mantener figuras profesionales. En este modelo la asistencia asume un idioma familiar, pero, como han evidenciado algunos estudios (Caradec, 1996; Déchaux, 1996; De Ridder y Legrand, 1996), esto crea una situación bastante contradictoria en la que las figuras de ayuda externa a la familia son llamadas a ser al mismo tiempo profesionales e íntimas. Además, como hemos anteriormente evidenciado haciendo referencia a Sahlins (1965; 2013), el tipo de transacciones que estas figuras ponen en acto pueden ser consideradas al mismo tiempo pertenecientes al ámbito de lo familiar o de lo económico, creando por tanto confusión sobre la distancia y el rango parental. La figura au pair en Francia meridional refleja todas estas tensiones, y por esto, nos ayuda a elaborar una crítica de la familia como matriz ideológica de un lenguaje social que regula las relaciones de asistencia y cuidado, así como a replantearnos nuestras ideas y definiciones de lazos fuertes y de familia en el contexto sur-europeo.

\section{Referencias bibliográficas}

Allan, Graham (2008). "Flexibility, friendship and family". Personal Relationships, 15: 1-16. Allen, Katherine R.; Blieszner, Rosemary; Roberto, Karen A. (2011). "Perspectives on Extended Family and Fictive Kin in the Later Years: Strategies and Meanings of Kin Reinterpretation”. Journal of Family Issues, 32, 9: 1156-1177.

Anderson, Bridget (2007). "A Very Private Business. Exploring The Demand for Migrant Domestic Workers”. European Journal of Women's Studies, 14, 3:247-264.

Attias-Donfut, Claudine; Segalen, Martine (1998). Grands-parents, la famille à travers les générations. Paris: Odile Jacob.

Attias-Donfut, Claudine; Lapierre, Nicole; Segalen, Martine (2002). Le nouvel esprit de famille. Paris: Odile Jacob. 
Baldassar, Loretta; Ferrero, Laura; Portis, Lucia (2017). "“More like a daughter than an employee': the kinning process between paid careers, their elderly employers and their extended families". Identities: Global Studies in Culture and Power, 24, 5: 524-541.

Bikova, Mariya (2008). A Family Member or a Family Servant? Why Norvegian Families Hire Au Pairs. A Qualitative Stud. Masteroppgave: Sosiologisk Institutt, Universitetet i Bergen.

Búriková, Zuzana (2006). "The Embarrassment of Co-Presence: Au Pairs and their Rooms": Home Cultures, 3 (2): 99-122.

Búriková, Zuzana; Miller, Daniel (2010). Au pair. Cambridge: Polity.

Çanga, Julina (2015). Au-pair, the ambiguous persona. How does the au-pair scheme applies to EU and non-EU citizens in Norway? A Qualitative Comparative Study. Master Thesis, Faculty of Social Sciences, Norwegian University of Life Sciences.

Caradec, Vincent (1996). “L’aide-ménagère: une employée ou une amie?”, en J.-C. Kaufmann (dir.), Faire ou faire-faire? Famille et services, Rennes: Presses Universitaires de Rennes, 155-170.

Carsten, Janet (2000). "Introduction: cultures of relatedness", en Id. (Ed.), Cultures of Relatedness. New Approaches to the Study of Kinship. Cambridge: Cambridge University Press.

Carsten, Janet (2004). After Kinship. Cambridge: Cambridge University Press.

Chatters, Linda M.; Taylor, Robert J.; Jayakody, Rukmalie (1994). "Fictive Kinship Relations in Black Extended Families". Journal of Comparative Family Studies, 25, 3: 297-312.

Cox, Rosie (2007). “The Au Pair Body: Sex Object, Sister or Student?” European Journal of Women's Studies, 14, 3: 281-296.

Cox, Rosie; Narula, Rekha (2003). "Playing Happy Families: roles and relationships in au pair employing households in London, England". Gender, Place and Culture, 10, 4: 333-344.

Cuartas Villa, Lina (2014). "Jóvenes au pairs: figura invisible de la migración y el empleo doméstico internacional”, en S. Durin, M.E. Martínez de la O, S. Bastos (Coords.), Trabajadoras en la sombra. Dimensiones del servicio doméstico latinoamericano, México: CIESAS-EGTP-ITM, 563-588.

Déchaux, Jean-Hugues (1996). "Les services dans la parenté: fonctions, régulations, effets”, en J.-C. Kaufmann (dir.), Faire ou faire-faire? Famille et services, Rennes: Presses Universitaires de Rennes. 39-54.

De Ridder, Guido: Legrand, Claude (1996). "Distance professionnelle et intimité affective", en J.-C. Kaufmann (dir.), Faire ou faire-faire? Famille et services, Rennes: Presses Universitaires de Rennes, 127-140.

Durin, Severine (2014). Las múltiples fachadas de las au pairs: hermana mayor, niñera de planta y empleada doméstica. Au pairs latinoamericanas y familias huéspedes en Marsella, Francia, en S. Durin, M.E. Martínez de la O, S. Bastos (Coords.), Trabajadoras en la sombra. Dimensiones del servicio doméstico latinoamericano, México: CIESASEGTP-ITM, 509-534.

Durin, Severine (2015). "Ethnicity and the Au Pair Experience: Latin American Au Pairs in Marseille, France”, en R. Cox (Ed.), Au Pairs' Lives in Global Context. Sisters or Servants?. London: Palgrave-MacMillan, 155-169.

Ebaugh, Helen R.; Curry, Marry (2000). "Fictive Kin as Social Capital in New Immigrants Communities”. Sociological Perspectives, 43, 2: 189-209.

Eleb, Monique (1996). "La frontière mouvante entre vie privée et vie publique dans la maison”, en J.-C. Kaufmann (dir.), Faire ou faire-faire? Famille et services, Rennes: Presses Universitaires de Rennes, 173-182. 
Ehrenreich, Barbara; Hochschild, Arlie R. (Eds.), 2003. Global Woman: Nannies, Maids, and Sex Workers in the New Economy. London: Macmillan.

González Díez, Javier; Viazzo, Pier Paolo (2016). "El parentesco ficticio entre América Latina y Europa: estrategias de respuesta a la desparentalización en perspectiva comparada". Confluenze. Rivista di studi iberoamericani, 8, 1: 89-104.

Grilli, Simonetta (2008). "'Un nuovo spirito di famiglia'? Casa, famiglia e parentela nella Toscana meridionale", en A. Rosina, P.P. Viazzo (Eds.), Oltre le mura domestiche. Famiglia e legami intergenerazionali dall'Unità d'Italia ad oggi. Udine: Forum, 143-169.

Hess, Sabine; Puckhaber, Annette (2004). “'Big Sisters’ Are Better Domestic Servants? Comments on the Booming Au Pair Business". Feminist Review, 77: 65-78.

INSEE (2012). Femmes et hommes. Regards sur la parité. Paris: Institut National de la Statistique et des Etudes Economiques - I.N.S.E.E.

Jelm, Erin (2010). Fictive Kinship and Acquaintance Networks as Sources of Support and Social Capital for Mexican Transmigrants in South Bend. Institute for Latino Studies, Student Research Brief 2.3.

Karner, Tracy (1998). "Professional Caring: Homecare Workers as Fictive Kin". Journal of Ageing Studies, 12, 1: 69-82.

Kaufmann, Jean-Claude (dir.), 1996. Faire ou faire-faire? Famille et services. Rennes: Presses Universitaires de Rennes.

Macdonald, Cameron L. (2010). Shadow mothers: Nannies, au pairs, and the micropolitics of mothering, Berkeley: University of California Press.

Martin, Claude (1996). "Solidarités familiales: débat scientifique, enjeu politique”, en J.-C. Kaufmann (dir.), Faire ou faire-faire? Famille et services. Rennes: Presses Universitaires de Rennes, 55-77.

Meillassoux, Claude (2000). "Parler parenté". L'Homme, 153: 153-164

Mellini, Laura; Yodanis, Carrie; Godenzi, Alberto (2007): “'On Par'? The Role of the Au Pair in Switzerland and France". European Societies, 9, 1: 45-64.

Moschion, Julie (2009). "Offre de travail des mères en France: l'effet causal du passage de deux à trois enfants". Economie et statistique, 422, 1: 51-78.

Muñoz Rodríguez, David (2014). "Criades globals: noves formes de precarització de les joves universitàries en situació d'au pair”. Quaderns de ciències socials, 28: 3-29.

Muraco, Anna (2006). "Fictive Kin Ties between Cross-Gender, Different Sexual Orientation Friends". Journal of Marriage and Family, 68, 5: 1313-1325.

Naldini, Manuela; Jurado, Teresa (2008). "Famiglia e Welfare: il modello sud-europeo", en A. Rosina, P.P. Viazzo (Eds.), Oltre le mura domestiche. Famiglia e legami intergenerazionali dall'Unità d'Italia ad oggi. Udine: Forum, 29-43.

Nelson, Margaret K. (2014). "Wither Fictive Kin? Or, What's in a Name?". Journal of Family Issues, 35, 2: 201-222.

Newcombe, Emma (2004). "Temporary migration to the UK as an 'Au Pair': Cultural exchange or reproductive labour?”. Sussex Migration Working Paper, 21.

Parreñas, Rhacel S. (2001). Servants of Globalization. Migration and Domestic Work. Standford: Standford University Press.

Piasere, Leonardo; Solinas, Pier Giorgio (1998). Le culture della parentela e l'esogamia perfetta. Roma: CISU.

Pitrou, Agnès (1996). "Le mythe de la famille et du familial”, en J.-C. Kaufmann (dir.), Faire ou faire-faire? Famille et services. Rennes: Presses Universitaires de Rennes, 25-38.

Pitt Rivers, Julian (1968). "Pseudo-kinship", en D.S. Sills (Ed.), International Encyclopedia of Social Sciences, 8: 408-413. 
Régnier-Lollier, Arnaud (Ed.), 2015. The Contemporary Family in France: Partnership Trajectories and Domestic Organization. London: Springer.

Reher, David S. (1998). "Family Ties in Western Europe: Persistent Contrasts". Population and Development Review, 24, 2: 203-234.

Remotti, Francesco (2008). Contro natura. Una lettera al papa. Roma-Bari: Laterza.

Sahlins, Marshall (1965). "On the Sociology of Primitive Exchange", en M. Banton (Ed.), The Relevance of Models for Social Anthropolog. London: Routledge, 139-235.

Sahlins, Marshall (2013). What Kinship Is - And Is Not. Chicago: University of Chicago Press.

Segalen, Martine; Martial, Agnes (2013). Sociologie de la famille. Paris: Armand Colin.

Sarti, Raffaella (1999). Vita di casa: abitare, mangiare, vestire nell'Europa moderna. RomaBari: Laterza.

Simpson, Bob (1994). "Bringing the 'Unclear' Family in the Focus: Divorce and Re-Marriage in Contemporary Britain”. Man, 39, 4: 831-851.

Solinas, Pier Giorgio (2004). L'acqua strangia. Il declino della parentela nella società complessa. Milano: Franco Angeli.

Viazzo, Pier Paolo, González Díez, Javier (2016). "Les 'nouvelles formes de famille en Italie'. Entre convergences morphologiques et persistances culturelles". Ethnologie française, 47, 2: 219-228.

Voorpostel, Marieke (2013). "Just Like Family: Fictive Kin Relationships in the Netherlands". Journals of Gerontology, Series B: Psychological Sciences and Social Sciences, 68, 5: 816-824.

Zanotelli, Francesco (2010). "Fare, disfare, moltiplicare. La produzione della parentela tra residenzialità, filiazione e cura", en S. Grilli, F. Zanotelli (Eds.), Scelte di famiglia. Tendenze della parentela nella società contemporanea, Pisa: ETS, 142-164. 